\title{
Calpain-Mediated Proteolysis of Talin and FAK Regulates Adhesion Dynamics Necessary for Axon Guidance
}

\author{
Patrick C. Kerstein, ${ }^{1,2}$ Kevin M. Patel, ${ }^{1}$ and $\mathbb{0}$ Timothy M. Gomez ${ }^{1,2}$ \\ ${ }^{1}$ Department of Neuroscience and ${ }^{2}$ Neuroscience Training Program, University of Wisconsin, Madison, Wisconsin 53705
}

Guidance of axons to their proper synaptic target sites requires spatially and temporally precise modulation of biochemical signals within growth cones. Ionic calcium $\left(\mathrm{Ca}^{2+}\right)$ is an essential signal for axon guidance that mediates opposing effects on growth cone motility. The diverse effects of $\mathrm{Ca}^{2+}$ arise from the precise localization of $\mathrm{Ca}^{2+}$ signals into microdomains containing specific Ca ${ }^{2+}$ effectors. For example, differences in the mechanical and chemical composition of the underlying substrata elicit local $\mathrm{Ca}^{2+}$ signals within growth cone filopodia that regulate axon guidance through activation of the protease calpain. However, how calpain regulates growth cone motility remains unclear. Here, we identify the adhesion proteins talin and focal adhesion kinase (FAK) as proteolytic targets of calpain in Xenopus laevis spinal cord neurons both in vivo and in vitro. Inhibition of calpain increases the localization of endogenous adhesion signaling to growth cone filopodia. Using live cell microscopy and specific calpain-resistant point-mutants of talin (L432G) and FAK (V744G), we find that calpain inhibits paxillin-based adhesion assembly through cleavage of talin and FAK, and adhesion disassembly through cleavage of FAK. Blocking calpain cleavage of talin and FAK inhibits repulsive turning from focal uncaging of $\mathrm{Ca}^{2+}$ within filopodia. In addition, blocking calpain cleavage of talin and FAK in vivo promotes Rohon-Beard peripheral axon extension into the skin. These data demonstrate that filopodial $\mathrm{Ca}^{2+}$ signals regulate axon outgrowth and guidance through calpain regulation of adhesion dynamics through specific cleavage of talin and FAK.

Key words: extracellular matrix; filopodia; integrin; paxillin; Xenopus

Significance Statement

The proper formation of neuronal networks requires accurate guidance of axons and dendrites during development by motile structures known as growth cones. Understanding the intracellular signaling mechanisms that govern growth cone motility will clarify how the nervous system develops and regenerates, and may identify areas of therapeutic intervention in disease or injury. One important signal that controls growth cones is that of local $\mathrm{Ca}^{2+}$ transients, which control the rate and direction of axon outgrowth. We demonstrate here that $\mathrm{Ca}^{2+}$-dependent inhibition axon outgrowth and guidance is mediated by calpain proteolysis of the adhesion proteins talin and focal adhesion kinase. Our findings provide mechanistic insight into $\mathrm{Ca}^{2+} / \mathrm{calpain}^{2}$ egulation of growth cone motility and axon guidance during neuronal development.

\section{Introduction}

Development of the nervous system requires precise spatial and temporal guidance of axons to form a functional neural circuit.

\footnotetext{
Received Aug. 31, 2016; revised Dec. 21, 2016; accepted Dec. 29, 2016.

Author contributions: P.C.K. and T.M.G. designed research; P.C.K., K.M.P., and T.M.G. performed research; P.C.K. and K.M.P. analyzed data; P.C.K. and T.M.G. wrote the paper.

This work was supported by National Institutes of Health (NIH) Grants R01-NS-099405 and R21-NS-088477 (to T.M.G.); NIH Grant F31-NS-074732 (to P.C.K.); and NIH Grant T32-GM-007507 (to the Neuroscience Training Program). We thank the members of the Gomez laboratory for helpful comments on this manuscript. We also thank Dr. Sucheta Kulkarni for the calpain construct, Dr. Alan F. Horwitz for the paxillin-GFP construct, Dr. Michael Davidson for the paxillin-tdTomato, and Dr. Anna Huttenlocher for the talin and FAK constructs.

The authors declare no competing financial interests.

Correspondence should be addressed to Timothy M. Gomez, University of Wisconsin, School of Medicine and Public Health, 5505 WIMR, 1111 Highland Avenue, Madison, WI 53705. E-mail: tmgomez@wisc.edu.

P.C. Kerstein's present address: Vollum Institute, Oregon Health \& Science University, Portland, 0 R 97239.

DOI:10.1523/JNEUROSCI.2769-16.2016

Copyright $\odot 2017$ the authors $\quad 0270-6474 / 17 / 371568-13 \$ 15.00 / 0$
}

An essential component of extending axons is a sensory and motile structure at the tip of the axon known as the growth cone. Studies have shown that growth cone motility is controlled in part through polymerization and depolymerization of two cytoskeleton components, actin filaments and microtubules (Lowery and Van Vactor, 2009; Dent et al., 2011). Less well understood are growth cone adhesion complexes, which link with the cytoskeleton to stabilize cell protrusions and provide the necessary traction for growth cone advance and turning (Kerstein et al., 2015; Nichol et al., 2016). Growth cones form small and transient adhesions known as point contact adhesions that are dynamically controlled by axon guidance cues (Myers et al., 2011; Vitriol and Zheng, 2012). A key component to adhesion-based guidance of axons is adhesion cycling. For example, attractive cues such as BDNF increase the formation and turnover of integrin-based 
adhesions (Myers and Gomez, 2011). Conversely, repulsive cues, such as Sema3A, EphrinA1, and Slit2, reduce the formation of adhesions and often stabilize existing adhesions to inhibit axon outgrowth or induce repulsive turning (Woo and Gomez, 2006; Bechara et al., 2008; Woo et al., 2009; Myers et al., 2012). Adhesion assembly and turnover are regulated by signaling through the kinases Src, focal adhesion kinase (FAK), and p21-associated kinase (Robles and Gomez, 2006; Woo and Gomez, 2006; Woo et al., 2009; Myers and Gomez, 2011; Santiago-Medina et al., 2013). While guidance cue receptors mediate some direct control over these kinases, it remains unclear whether additional biochemical signals mediate control over adhesion dynamics in growth cones.

In migrating cells, ionic calcium $\left(\mathrm{Ca}^{2+}\right)$ is a critical biochemical regulator of cell polarity and motility (Wei et al., 2009). One primary mechanism by which $\mathrm{Ca}^{2+}$ guides cell migration is through the $\mathrm{Ca}^{2+}$-dependent cysteine protease calpain. Previous work suggests that $\mathrm{Ca}^{2+}$ and calpain regulate cell migration though the proteolytic degradation of cytoskeleton-integrin linkages (Huttenlocher et al., 1997). Additional studies identified talin, an integrin adaptor protein, and FAK, an adhesion signaling protein, as two principal targets of calpain proteolysis, which, when cleaved, inhibit adhesion dynamics (Franco et al., 2004; Chan et al., 2010). Interestingly, recent studies suggest that neuronal morphology and growth cone motility are also regulated by calpain activity. In developing neurons, substratum adhesivity regulates local filopodial $\mathrm{Ca}^{2+}$ transients within growth cones (Gomez et al., 2001) and inhibits axon outgrowth by calpaindependent disruption of tyrosine kinase signaling and talin localization (Robles et al., 2003; Kerstein et al., 2013). While previous studies have demonstrated a clear role for calpain activity in cell migration and axon development, the precise targets and underlying mechanisms of calpain in the developing nervous system remain elusive.

In this study, we investigated two possible targets of calpain proteoylsis within point contact adhesions talin and FAK. While calpain has previously been shown to cleave both talin and FAK in HEK293 cells in vitro (Franco et al., 2004; Chan et al., 2010); here we show that cleavage of talin and FAK occurs naturally within the developing Xenopus spinal cord. Functionally, we show that calpain activation reduces adhesion signaling within growth cone filopodia and inhibits point contact adhesion dynamics, by preventing adhesion formation and stabilizing existing adhesions. Moreover, expressing calpain-resistant point mutants of talin (L432G) or FAK (V744G) in growth cones modulates adhesion dynamics. Finally, inhibiting proteolysis of these key adhesion proteins blocks calpain-dependent growth cone turning in vitro and axon extension in vivo. While many studies have suggested an important role for $\mathrm{Ca}^{2+}$ signaling in growth cones, few have identified downstream $\mathrm{Ca}^{2+}$ effectors that regulate the cytoskeleton to control axon guidance. This study describes a precise mechanism for $\mathrm{Ca}^{2+}$ control over adhesion turnover to regulate axon guidance.

\section{Materials and Methods}

Expression constructs. When needed expression constructs were subcloned into the Xenopus-preferred pCS2 vector (provided by Dave Turner, University of Michigan, Ann Arbor, MI). Human calpain1 H272A was provided by S. Kulkarni (Cleveland Clinic Foundation, Cleveland, $\mathrm{OH}$ ) and was subcloned into pCS2 C-GFP (Kulkarni et al., 1999). Chick paxillin-eGFP (plasmid \#15233, Addgene; Laukaitis et al., 2001) was provided by A.F. Horwitz (University of Virginia, Charlottesville, VA) and subcloned into pCS2. Chick paxillin-tdTomato (plasmid \#58123, Addgene) was provided by M. Davidson (National Magnetic
Field Laboratory, Florida State University, Tallahassee, FL). Mouse eGFP-talin L432G (plasmid \#26725, Addgene; Franco et al., 2004), eGFP-FAK V744G, and TagRFP-FAK V744G (Chan et al., 2010) were provided by A. Huttenlocher (University of Wisconsin, Madison, WI). Male and female Xenopus laevis embryos (Nasco) were obtained and staged as described previously (Nieuwkoop and Faber, 1994; Gómez et al., 2003). For experiments requiring plasmid expression, two to three blastomeres of eight-cell-stage embryos were injected with 50-75 pg of DNA constructs. For in vivo skin prep experiments, single dark blastomeres of eight-cell-stage embryos were injected. Embryos that appeared grossly normal $24 \mathrm{~h}$ postfertilization (hpf) were used to make spinal cord explant cultures. Neural tube explant cultures containing neurons were prepared in a modified Ringer's solution, as described previously (Gómez et al., 2003). Aminoglycoside antibiotics (AGAs), gentamicin and streptomycin, are present in our culture media at a concentration of 100 $\mu \mathrm{M}$ each for antimicrobial purposes. Explants were plated onto acidwashed coverslips coated with $25 \mu \mathrm{g} / \mathrm{ml}$ laminin (LN; Sigma-Aldrich). Cultures were imaged or fixed 16-24 h after plating.

Image acquisition and analysis. For fixed fluorescence microscopy, images were acquired using a $60 \times / 1.45$ numerical aperture (NA) or a $10 \times /$ 0.3 NA objective lens using a Olympus Fluoview 500 laser-scanning confocal system mounted on an AX-70 upright microscope for in vitro and in vivo experiments, respectively. Olympus Fluoview software was used for image acquisition (RRID: SRC_014215). On the confocal system, fixed samples used for immunocytochemistry experiments were imaged with a $2.5 \times$ zoom (pixel size, $165 \mathrm{~nm}$ ). Measurements of filopodia immunofluorescence intensity of talin, vinculin, pY397 FAK, pY99, and pY188 paxillin were made by first selecting the perimeter of growth cones from thresholded filamentous actin (F-actin)-labeled images based on intensity to exclude background. Furthermore, the centers of growth cones were removed manually from the selection mask to measure filopodia using only FIJI open-source software (RRID: SRC_002285). These user-defined regions were then used to measure the average pixel intensity of immunolabeling within nonthresholded growth cone filopodia. For display purposes, some images were pseudocolored using FIJI lookup tables. For live cell adhesion fluorescence microscopy, images were captured using a $100 \times / 1.5 \mathrm{NA}$ total internal reflection fluorescence (TIRF) objective lens on a Nikon TIRF microscope with a CoolSNAP HQ2 CCD camera (Photometrics). Nikon MetaMorph software was used for image acquisition (RRID: SRC_002368). For all live cell adhesion experiments, explant cultures were sealed within perfusion chambers, as described previously (Gómez et al., 2003), to allow the rapid exchange of solutions. Time-lapse images of paxillin-GFP or paxillintdTomato puncta were captured at $5 \mathrm{~s}$ intervals for $10 \mathrm{~min}$ before and after the addition of $1 \mu \mathrm{M}$ calpastatin peptide inhibitor (CPI; Calbiochem) or the removal of AGAs. Only growth cones that did not collapse before or after pharmacological treatment were analyzed. Images were analyzed off-line using FIJI. Point contacts were identified as discrete puncta containing paxillin-GFP or paxillin-tdTomato that were at least two times brighter than the surrounding background and remained stationary for at least $30 \mathrm{~s}$ (Woo and Gomez, 2006). For all figures, images were processed in Photoshop (Adobe Systems; RRID: SCR_014199) as follows: brightness levels were adjusted, an unsharp mask routine was applied to improve edge detection, and the images were converted to an 8 bit depth and cropped.

Dynamic adhesion maps. Dynamic adhesion map images were prepared from image stacks using FIJI, as detailed previously (SantiagoMedina et al., 2011). Briefly, an image stabilization algorithm was applied if necessary, and to improve edge detection an unsharp mask routine was applied, followed by thresholding to highlight the puncta of interest. Next, an 8 bit binary filter was applied to equalize point contact intensities. Binary images were dilated by 1 pixel to improve the detection of small point contact adhesions. Image stacks were then converted to 16 bit and summed so that intensity provides a measure of pixel lifetime. Final images were contrast enhanced and pseudocolored.

Caged $\mathrm{Ca}^{2+}$ experiments. For focal $\mathrm{Ca}^{2+}$ uncaging experiments, a $100 \times / 1.40$ NA objection lens was used on an Olympus Fluoview 500 laser-scanning confocal system mounted on an AX-70 upright microscope. Neurons on LN were loaded for 45 min with $4 \mu \mathrm{M}$ NP-EGTA AM 
(Invitrogen) and photoactivated with $360 \pm 25 \mathrm{~nm}$ light from a mercury lamp, as described previously (Robles et al., 2003). Growth cone filopodia loaded with NP-EGTA and Fluo-4 (Invitrogen) were used to calibrate the UV pulse conditions to match the amplitude and kinetics of natural filopodial $\mathrm{Ca}^{2+}$ transients, as described previously (Gomez et al., 2001). During turning assays, the leading edge of motile growth cones was positioned $5 \mu \mathrm{m}$ from the region of UV light. Differential interference contrast or GFP fluorescence images were acquired every $15 \mathrm{~s}$. Only growth cones that advanced $10 \mu \mathrm{m}$ during the imaging time period were used for off-line analysis of axon turning angles using FIJI software.

In vivo skin preparation. Rohon-Beard (RB) peripheral axons were visualized in stage $25-26$ embryos by isolating the dorsal skin by removing the spinal cord and lateral somites. The skin whole-mounts were incubated with 1:500 anti-GFP (Abcam; RRID: AB_305564) and 1:500 anti-HNK-1 antibody (NCAM; Sigma-Aldrich; RRID: AB_1078474) to label RB axons. Multiple confocal fields of view of a single preparation were stitched together using the MosaicJ plugin within the FIJI software (Thévenaz and Unser, 2007). For analysis of RB peripheral axon outgrowth, the length of the longest RB axon was measured within $100 \mu \mathrm{m}$ segments and compared between the injected and uninfected sides of the skin, as described previously (Robles and Gomez, 2006; Moon and Gomez, 2010).

Reverse transcription-PCR. For reverse transcription (RT)-PCR experiments, cDNA libraries were made from mRNA isolated from stage 22 Xenopus spinal cords using a TRIzol (Invitrogen)-based extraction protocol, and reverse transcription was performed using ImProm-II Reverse Transcription System (Promega). Primers for calpain1, calpain2, calpainSS1, and $\beta$ II-tubulin were designed from X. laevis-specific mRNA sequences obtained from Xenbase and National Center for Biotechnology Information. PCR experiments were completed using DreamTaq Polymerase (Thermo Scientific).

Immunocytochemistry and Western blots. For immunocytochemistry experiments, spinal neuron cultures were fixed in 4\% paraformaldehyde in Krebs sucrose fixative (Dent and Meiri, 1992), permeabilized with $0.1 \%$ Triton X-100, and blocked in $1.0 \%$ fish gelatin in calciummagnesium-free PBS for $1 \mathrm{~h}$ at room temperature. Primary antibodies were used at the following dilutions in blocking solution: 1:500 talin (Thermo Fisher; RRID: AB_2204008), 1:500 vinculin (Sigma-Aldrich; RRID: AB_477629), 1:500 pY397 FAK (Thermo Fisher; RRID: AB_2533701), 1:500 pY99 (Santa Cruz Biotechnology; RRID: AB_628123), and 1:500 phospho-Y118 paxillin (Thermo Fisher; RRID: AB_2533733) antibodies. Alexa Fluor-conjugated secondary antibodies were purchased from Invitrogen and used at 1:250 in blocking solution (RRID: AB_141514, RRID: AB_143165, RRID: AB_141370, RRID: AB_143051). Included with secondary antibodies was Alexa Fluor 546 and Alexa Fluor 647 phalloidin (1:100; Invitrogen, RRID: AB_2572408, RRID: AB_2620155) to label F-actin. For Western blots, talin, FAK, and $\beta$ I-II tubulin were blotted from total protein extracts from stage 22-23 embryo spinal cords that were treated with either $1 \mu \mathrm{M} \mathrm{CPI}$ or $0.1 \%$ DMSO for $30 \mathrm{~min}$ before protein isolation. Spinal cord lysates were run on a Novex NuPAGE SDS-PAGE gel system (Invitrogen). Talin (1:1000; Thermo Fisher; RRID: AB_2204008), FAK (1:500; Cell Signaling Technology; RRID: AB_10694098), and $\beta$ I-II tubulin (1:1000; Sigma; RRID: AB_261795) primary antibodies were used for immunoblotting and were visualized with horseradish peroxidase-conjugated secondary antibodies (1:5000; Jackson ImmunoResearch; RRID: AB_10015289, RRID: AB_2313597). The blots were developed and visualized using enhanced chemiluminescence (Thermo Scientific).

$t$-BOC-L-leucyl-L-methionine amide proteolysis assay. Xenopus neurons were incubated with $10 \mu \mathrm{M}$ 7-amino-4-chloro-methylcoumarin, t-BOC-L-leucyl-L-methionine amide (t-BOC; catalog \#A6520, Invitrogen) in $0.01 \%$ pluronic acid $/ 0.1 \%$ DMSO in MR. Coumarin fluorescence was monitored using a $40 \times$ oil-immersion objective on an inverted Nikon Eclipse TE2000-E microscope equipped with a Prior Lumen 200PRO halide lamp filtered through $350 \pm 25 \mathrm{~nm}$ excitation and $460 \pm 25 \mathrm{~nm}$ emission filters (Chroma Technology). Coumarin fluorescence intensity within growth cones was captured at 0 and $10 \mathrm{~min}$ after t-BOC addition.

Statistical analysis. For all datasets, the variance is reported as \pm SEM. Each dataset was first tested for normality. Analysis between two groups was completed by using an unpaired Student's $t$ test (parametric) or a Mann-Whitney $U$ test (nonparametric). For analysis among more than two groups, either a one-way ANOVA with Tukey's multiplecomparison test (parametric) or a Kruskal-Wallis with Dunn's multiplecomparison test (nonparametric) was used. GraphPad Prism Software (RRID: SRC_002798) was used for statistical significance tests.

\section{Results \\ Calpain-mediated proteolysis of talin and FAK in the developing spinal cord and in growth cones}

To investigate the targets of calpain-mediated proteolysis in developing neurons, we first confirmed the presence of conventional calpain transcripts in developing Xenopus spinal cords expressed by RT-PCR from stage 22 spinal cord mRNA. We found that the proteolytic subunits calpain 1 ( $\mu$-calpain) and calpain2 (m-calpain), and the regulatory subunit calpain small subunit 1 (calpainSS1, calpain4) are expressed at this stage of robust axon extension (Fig. 1a). Next, we took a candidate approach by selecting likely calpain targets identified in migrating nonneuronal cells. The migration of fibroblasts is disrupted by perturbations in calpain function through proteolysis and degradation of adhesion and cytoskeletal components (Huttenlocher et al., 1997). Two of the main targets within adhesion complexes are the scaffolding protein talin and the tyrosine kinase FAK (Franco et al., 2004; Chan et al., 2010). In previous studies, calpain-mediated proteolysis of talin and FAK was analyzed in human cell lines. Therefore, we first examined whether the calpain cleavage sites of talin and FAK were conserved in $X$. laevis and then tested whether cleavage of these proteins occurs in primary neurons (Fig. 1b,c). To assess whether talin and FAK were cleaved in the developing spinal cord, we collected stage 22 spinal cord lysates. At this stage, neurons are actively extending axons in the spinal cord (Robles and Gomez, 2006; Moon and Gomez, 2010). By Western blot, talin appears as a $230 \mathrm{kDa}$ fulllength band, a $190 \mathrm{kDa}$ rod fragment band, and a $120 \mathrm{kDa}$ Vinculin-Actin-Dimerization domain fragment (Fig. 1d). All bands appear as doublets, since this antibody recognizes both isoforms of talin. Importantly, we know these talin fragments are due to calpain cleavage since the inhibition of calpain by incubating the spinal cords for $30 \mathrm{~min}$ in $1 \mu \mathrm{M}$ CPI reduces talin cleavage by $\sim 70 \%$ relative to the DMSO control (Fig. $1 d$ ). Western blots labeled for FAK show a $116 \mathrm{kDa}$ full-length band and an $80 \mathrm{kDa}$ cleaved fragment, which are reduced by $\sim 60 \%$ relative to the control after inhibition of calpain (Fig. 1e). These data demonstrate that talin and FAK are cleaved by calpain in developing spinal cord lysates, suggesting a possible role for calpain during axon extension and pathfinding.

While analysis of spinal cord lysates demonstrates calpain cleavage of proteins in native conditions, they do not prove proteolysis occurs within growth cones. Therefore, we used immunocytochemistry for key adhesion proteins and phosphorylation sites to determine whether changes in calpain activity modulated adhesion protein localization and signaling in growth cones. We first assessed localization of three different adhesion proteins and phosphorylation sites that have previously been shown to localize to growth cone filopodia, as follows: talin; an active form of FAK (pY397 FAK); and a point contact adhesion marker pY118 paxillin (Robles and Gomez, 2006; Woo and Gomez, 2006; Kerstein et al., 2013; Fig. 2a,d,g). We quantified the immunofluorescence signal intensity within individual filopodia instead of entire growth cones, as dynamic $\mathrm{Ca}^{2+}$, calpain, and adhesion signals are most robust in filopodia (Robles et al., 2003; Kerstein et al., 2013). First, we inhibited calpain activity with $1 \mu \mathrm{M}$ CPI for $30 \mathrm{~min}$ 
a

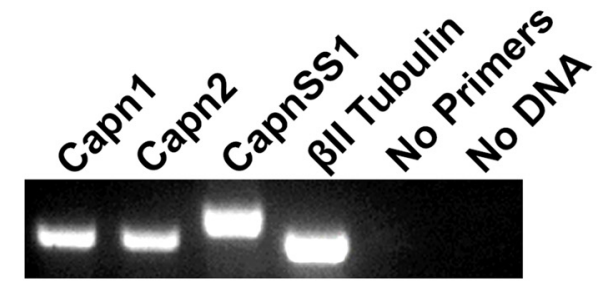

St. 22 Spinal Cord mRNA

b

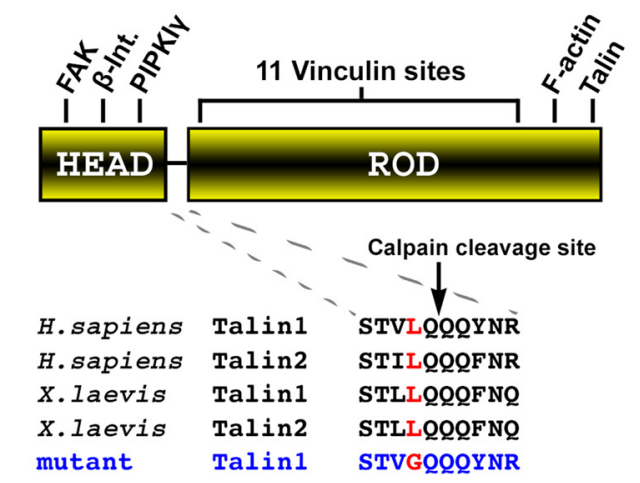

c FAK

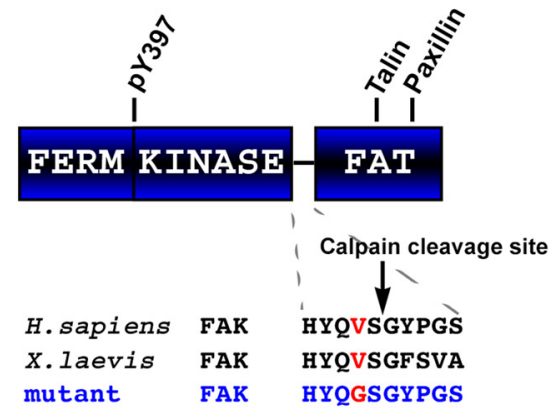

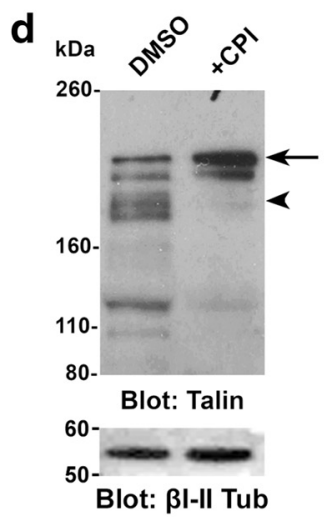
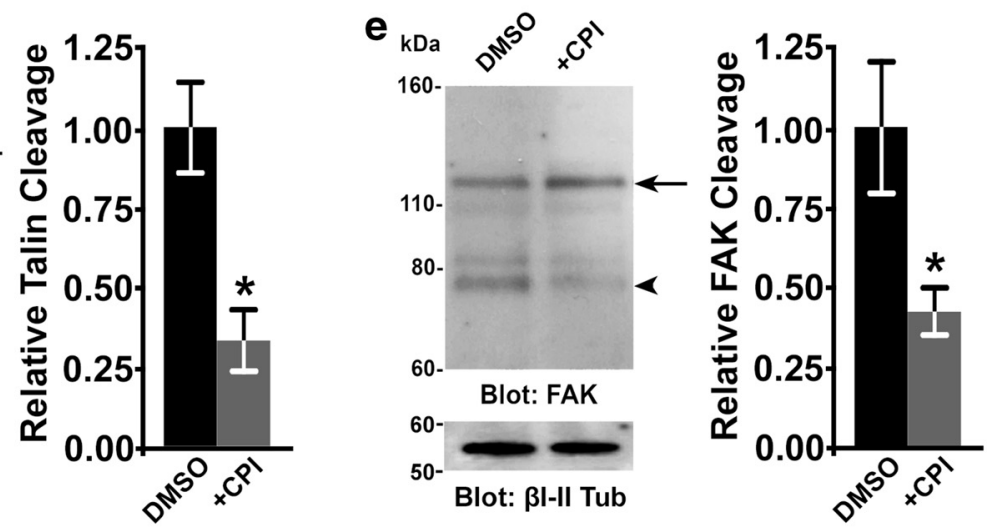

Figure 1. Talin and FAK are cleaved by calpain within the embryonic spinal cord. $\boldsymbol{a}$, RT-PCR amplification of mRNA transcripts of calpain1 (262 bp), calpain2 (262 bp), calpainSS1 (299 bp), and $\beta \|$ tubulin (251 bp) from stage 22 Xenopus spinal cords. $\boldsymbol{b}, \boldsymbol{c}$, Schematic domain organization of talin (b) and FAK (c) showing approximate location of protein interaction motifs and calpain cleavage sites. Below are human and Xenopus protein sequences of the calpain cleavage sites, which are highly conserved between species. $\boldsymbol{d}$, Immunoblots for talin from Xenopus neural tube lysates incubated in control media or media containing a calpain protease inhibitor ( $1 \mu \mathrm{m} \mathrm{CPI)} \mathrm{for} 30 \mathrm{~min}$. The full-length talin band is at $230 \mathrm{kDa}$ (arrow), and the cleaved talin is at $190 \mathrm{kDa}$ (arrowhead). An additional calpain-dependent fragment (Vinculin-Actin-Dimerization domain fragment) was found at $120 \mathrm{kDa}$ (not quantified). Note that all bands appear as doublets since the antibody recognizes both talin isoforms. The average talin cleavage in control and CPI (right) was quantified from the large isoform band intensities (190 kDa band/230 kDa) from multiple blots ( $n=7)$. $\boldsymbol{e}$, Immunoblots for FAK from Xenopus neural tube lysates incubated in control media or media containing a calpain protease inhibitor ( $1 \mu \mathrm{M}$ (PI) for 30 min. The full-length FAK band is at $116 \mathrm{kDa}$ (arrow), and cleaved FAK is at $80 \mathrm{kDa}$ (arrowhead). The average FAK cleavage in control and CPI (right) was quantified ( $80 \mathrm{kDa}$ band/116 kDa) from multiple blots $(n=6)$. ${ }^{*} p<0.05$, Mann-Whitney U test.

before fixation and found that talin, pY397 FAK, and pY118 paxillin all increased in fluorescence intensity within filopodia (Fig. $2 b, e, h, j)$. Next, we acutely activated calpain in a $\mathrm{Ca}^{2+}$-dependent manner by removing AGAs from the culture media 30 min before fixation. Previous studies have shown that AGAs, which are common cell culture antibiotics, also inhibit mechanosensitive ion channels (Kerstein et al., 2013). Furthermore, acute removal of AGAs elicits filopodial $\mathrm{Ca}^{2+}$ transients in a robust and reproducible manner. Removal of AGAs acts on growth cone motility through the disinhibition of mechanosensitive transient receptor potential canonical member 1 (TRPC1) channels that selectively and directly activate calpain through local $\mathrm{Ca}^{2+}$ influx (Kerstein et al., 2013). When we activated calpain through acute removal of AGAs for 30 min before fixation, we found a reduction in talin,
pY397 FAK, and pY118 paxillin fluorescence intensity in growth cone filopodia (Fig. $2 c, f, i, j)$. In addition, we found a similar calpain-dependent trend for the fluorescent intensity of other adhesions signaling markers, such as the talin binding protein vinculin and total tyrosine phosphorylation (Fig. $2 j$ ), which we have previously shown to be highly localized to point contact adhesions (Robles et al., 2005).

To support our pharmacological manipulations, we expressed a catalytically inactive calpain 1 (capn1-H272A) mutant in spinal neurons. Capn1-H272A has previously been shown to act as a dominant negative, as it competes with endogenous calpain 1 and calpain2 for binding to the regulatory and activating subunit, calpain small subunit 1 (Capns1, Capn4; Kulkarni et al., 1999). Importantly, capn1-H272A expression in Xenopus spinal cord 

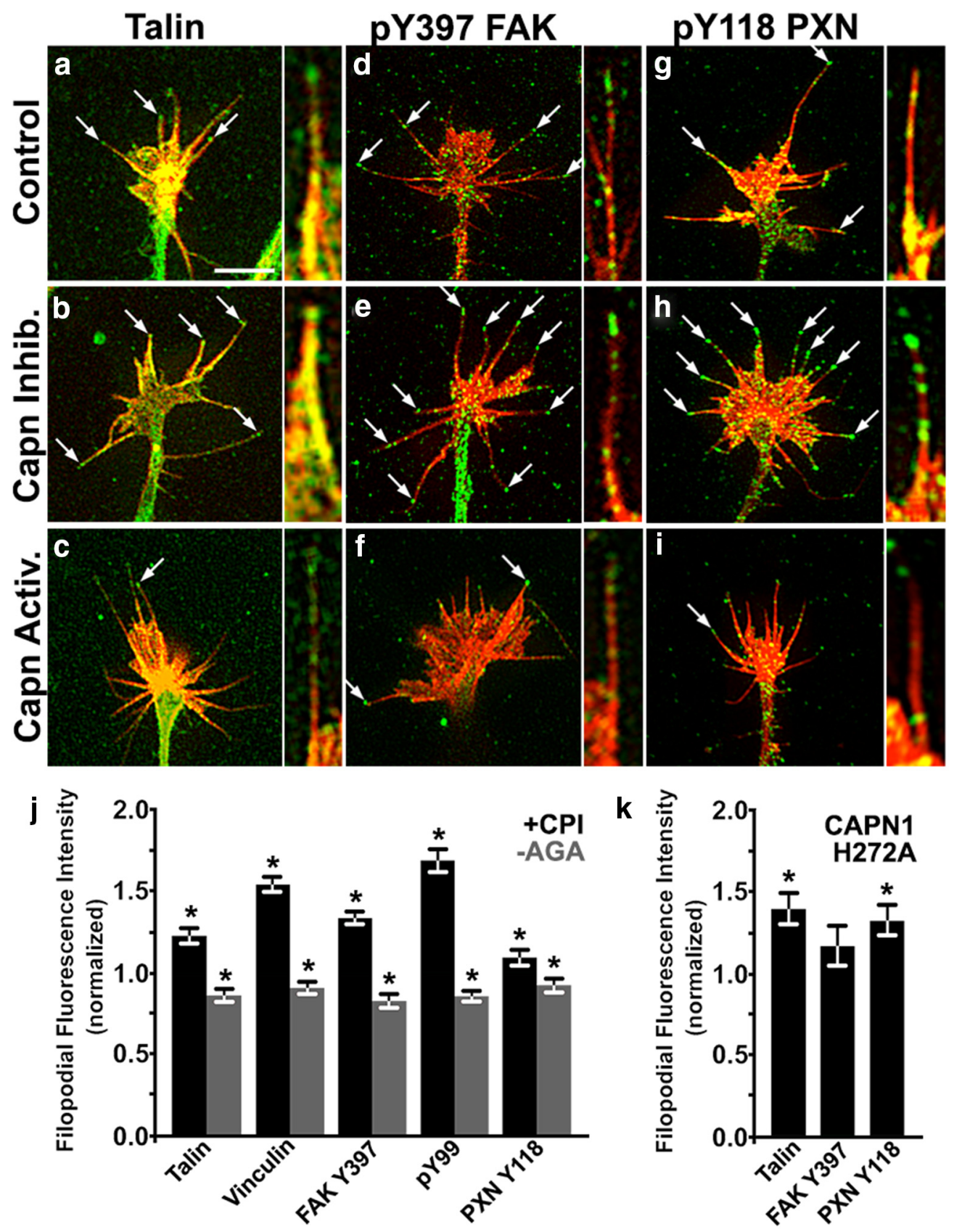

Figure 2. Calpain activity regulates adhesion protein localization and signaling in filopodia. $\boldsymbol{a}-\boldsymbol{i}$, Immunocytochemistry experiments were performed with cultured Xenopus spinal neurons plated on laminin. The fluorescence intensity within filopodia was quantified in the following three conditions: control, with $1 \mu \mathrm{m}$ CPI (calpain inhibition), or without AGAs (-AGAs, calpain activation). $\boldsymbol{a}-\boldsymbol{i}$, Representative images of growth cones (left) and filopodia (right) shown for selected adhesion proteins, as follows: talin ( $\boldsymbol{a}$ - $\boldsymbol{c}$ ), phospho-tyrosine 397 FAK (FAK Y397; $\boldsymbol{b}$ - $\boldsymbol{h}$ ), and phospho-tyrosine 118 paxillin (PXN Y118; $\mathbf{c}-\boldsymbol{i}$ ). Immunolabeled adhesion proteins are in green, and phallodin-labeled F-actin is in red. Arrows indicate localization to filopodial tips. $\boldsymbol{j}$, Quantification of filopodial fluorescence intensity of all adhesion markers tested, including vinculin and total phospho-tyrosine (pY99), with inhibition of calpain or $\mathrm{Ca}^{2+}$-dependent activation of calpain by removal of AGAs. $\boldsymbol{k}$, Quantification of filopodial fluorescence intensity of talin, FAK Y397, and PXN Y118 in growth cones expressing GFP-tagged dominant-negative calpain1 (H272A) normalized to cocultured wild-type neurons. Scale bar, $5 \mu \mathrm{m} . n>60$ growth cones and $n>3$ cultures for all conditions. ${ }^{*} p<0.05$, Mann-Whitney $U$ test.

neurons shows that it localizes to neuronal growth cones and reduces protease activity (data not shown). Growth cones expressing capn1-H272A exhibited a significantly higher fluorescence intensity of talin and pY118 paxillin in filopodia, consistent with pharmacological inhibition of calpain (Fig. 2k). However, while pY397 FAK showed a similar increase in filopodial localization, this increase was not statistically significant (Fig. $2 k$ ). These data suggest that calpain cleaves adhesion proteins within the developing spinal cord and within growth cone filopodia to reduce adhesion signaling, and therefore may regulate specific aspects of point contact adhesion dynamics in motile growth cones.

Calpain activity regulates point contact adhesion dynamics in neuronal growth cones

We precisely measure point contact assembly frequency and duration from live paxillin-GFP-expressing growth cones imaged at high temporal and spatial resolutions with TIRF microscopy (Woo et al., 2009; Myers and Gomez, 2011). Using this approach, we previously correlated the rate of point contact turnover with 

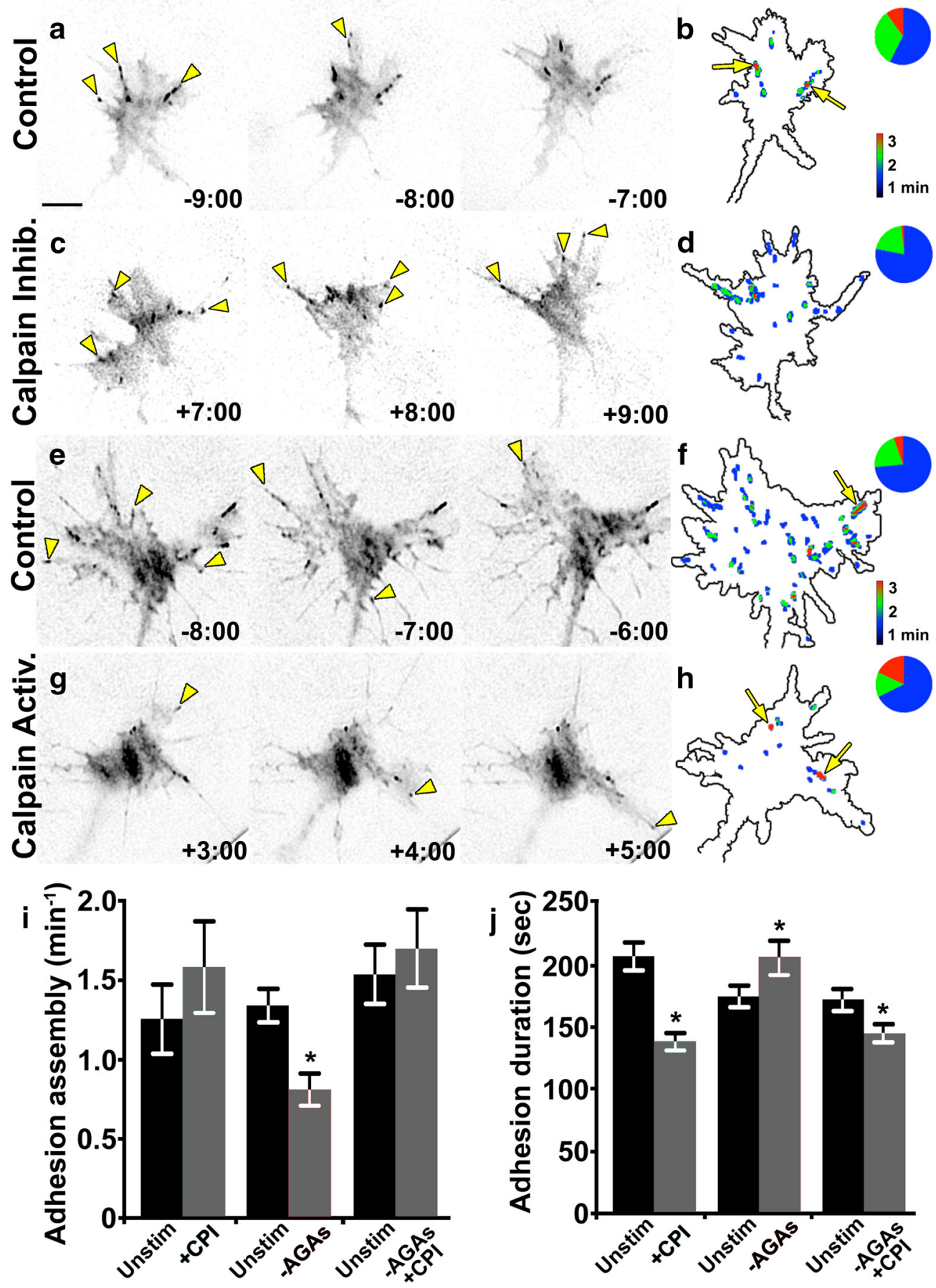

Figure 3. Calpain activity regulates adhesion dynamics in filopodia. $\boldsymbol{a}, \boldsymbol{c}$, Inverted contrast TIRF images of growth cones expressing paxillin-GFP displayed at 1 min intervals, over 10 min before (a) and after (c) the addition of CPI (calpain inhibition). $\boldsymbol{b}, \boldsymbol{d}$, Adhesion lifetime heat maps showing adhesion dynamics from the time points in $\boldsymbol{a}$ and $\boldsymbol{c}$, respectively. The inset pie charts demonstrate the proportion adhesions with the lifetime $0-1 \mathrm{~min}$ (blue), 1-2 $\mathrm{min}$ (green), and 2-3 min (red). Note that the inhibition of calpain reduces the number of stable adhesions (arrows) and increases the number new adhesions (arrowheads). $\boldsymbol{e}, \boldsymbol{g}$, Inverted contrast TIRF images of growth cones expressing paxillin-GFP displayed at 1 min intervals $(\boldsymbol{e})$ before and $(\boldsymbol{g})$ after the removal of AGAs (calpain activation). $\boldsymbol{f}, \boldsymbol{h}$, Adhesion lifetime heat maps showing adhesion dynamics from the time points in $\boldsymbol{e}$ and $\boldsymbol{g}$, respectively. Note that $\mathrm{Ca}^{2+}$-dependent activation of calpain reduces the formation of new adhesions (arrowheads) and stabilizes existing adhesions (arrows). $\boldsymbol{i}, \boldsymbol{j}$, Quantification of adhesion assembly (i) and duration (j) for calpain inhibition (+ CPI), activation ( $-\mathrm{AGAs}$ ), or the combined removal of AGAs with the addition of CPI. Scale bar, $5 \mu \mathrm{m} . n>100$ adhesions and $n>12$ growth cones for each condition. ${ }^{*} p<0.05$, Mann-Whitney $U$ test.

the rate of axon outgrowth (Woo and Gomez, 2006; Woo et al., 2009; Myers and Gomez, 2011). Since calpain inhibition increases axon outgrowth (Robles et al., 2003; Kerstein et al., 2013) and increases adhesion signaling within filopodia (Fig. 2), we hypothesized that calpain inhibition would promote point contact turnover. To test this hypothesis, we acquired time-lapse images of paxillin-GFP-expressing growth cones $10 \mathrm{~min}$ before and after applying $1 \mu \mathrm{M}$ CPI (Fig. $3 a, c$ ). We found that the inhibition of calpain caused little or no increase $(26.2 \%$; not significant) in point contact assembly (Fig. $3 a-d, i)$, but did cause a $33.1 \%$ reduction in point contact duration (Fig. $3 a-d, j$ ). Conversely, when we acutely activated calpain with the removal of AGAs, we found a $40.0 \%$ reduction in point contact assembly and a $19.4 \%$ increase in point contact adhesion duration (Fig. $3 e-j$ ). 


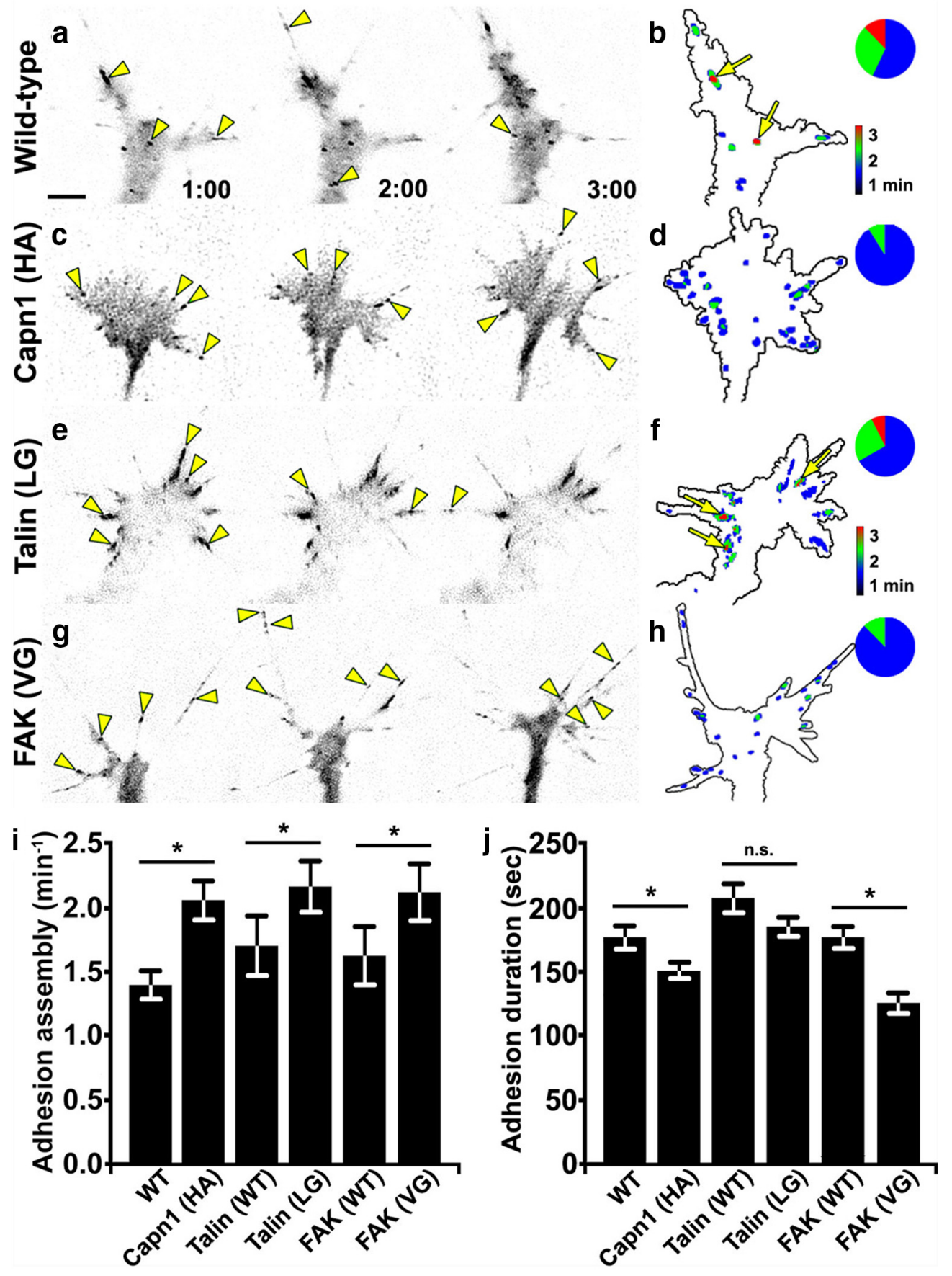

Figure 4. Calpain regulates adhesion dynamics through the cleavage of talin and FAK. $\boldsymbol{a}-\boldsymbol{h}$, Inverted contrast TIRF images of growth cones expressing paxillin-GFP or paxillin-tdTomato displayed every 1 min over a 3 min period in wild-type- $(\boldsymbol{a}, \boldsymbol{b})$, dominant-negative calpain1 (H272A; $\boldsymbol{c}, \boldsymbol{d})$, calpain-resistant talin- (L432G; $\boldsymbol{e}, \boldsymbol{f})$, or calpain-resistant FAK (V744G; $\boldsymbol{g}, \boldsymbol{h}$ )-expressing neurons. Arrowheads denote new adhesions formed. $\boldsymbol{b}, \boldsymbol{d}, \boldsymbol{f}, \boldsymbol{h}$, Adhesion lifetime heat maps exhibit adhesion dynamics from the corresponding time points. Arrows represent the stable adhesions that have a lifetime between 2 and $3 \mathrm{~min}$. The inset pie charts demonstrate the proportion of adhesions with lifetimes of $0-1$ min (blue), $1-2$ min (green), and 2-3 min (red). $\boldsymbol{i}, \boldsymbol{j}$, Quantification of adhesion formation (i) and duration (j) for wild-type-, capn1-H272A-, talin-WT-, talin-L432G-, FAK-WT-, and FAK-V744G-expressing growth cones. Statistical comparisons were made between wild-typeand capn1-H272A-, talin-WT- and talin-L432G-, and FAK-WT- and FAK-V744G-expressing growth cones. Scale bar, $5 \mu \mathrm{m} . n>100$ adhesions and $n>11$ growth cones for each condition. ${ }^{*} p<0.05$, Mann-Whitney $U$ test or Student's $t$ test.

Consistent with the activation of calpain, the effects of AGA removal were suppressed or reversed in the presence of CPI (Fig. 3i,j).

To validate our pharmacological findings, we coexpressed dominant-negative mCherry-capn1-H272A and paxillin-GFP in spinal neurons to assess growth cone adhesion dynamics during chronic calpain inhibition (Fig. 4c,d). In growth cones expressing capn1-H272A, we observed a $42.7 \%$ increase in adhesion assem- bly and a $14.4 \%$ decrease in adhesion duration compared with wild-type growth cones (Fig. $4 a-d, i, j)$, which are similar to observations with acute application of CPI (Fig. 3i,j). In addition, the activation of filopodial $\mathrm{Ca}^{2+}$ transients by AGA removal had no effect on adhesion dynamics in growth cones expressing dominant-negative calpain1 (Fig. 5). These data suggest that the inhibition of calpain activity increases adhesion cycling through an increase in point contact assembly and a decrease in adhesion 

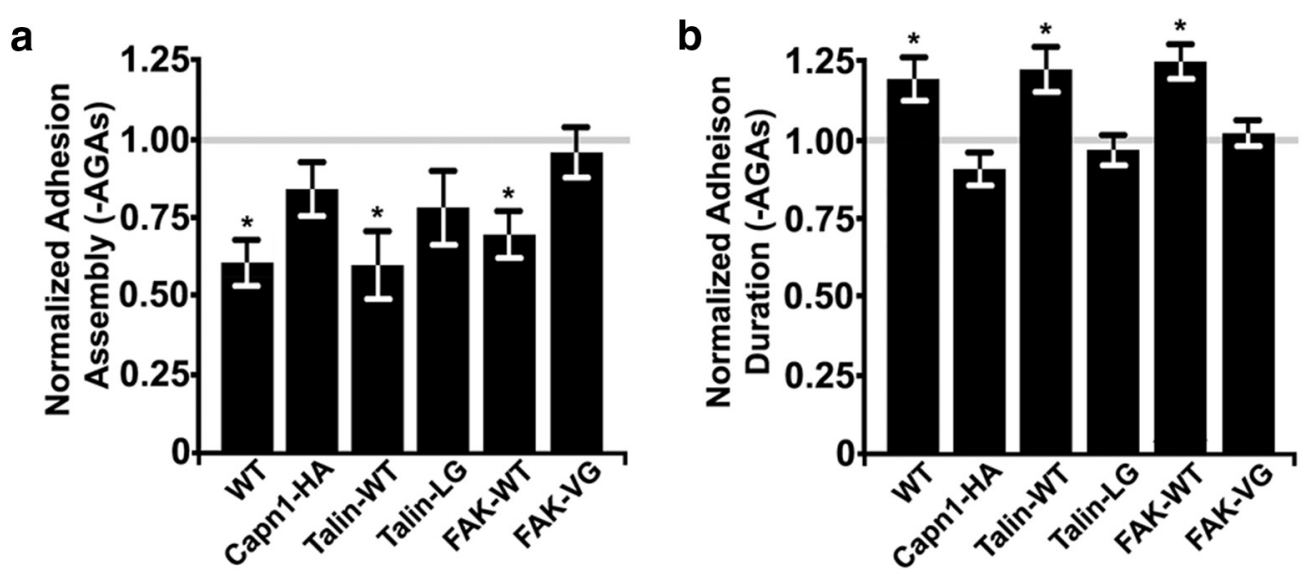

Figure 5. The effects of filopodial $\mathrm{Ca}^{2+}$ transients on adhesion dynamics are blocked by inhibition of calpain activity and cleavage of talin and FAK. $\boldsymbol{a}, \boldsymbol{b}$, Change in growth cone adhesion assembly rate $(\boldsymbol{a})$ and adhesion duration $(\boldsymbol{b})$ after disinhibition of filopodial $\mathrm{Ca}^{2+}$ transients by removing AGAs in wild-type-, capn1-H272A-, talin-WT-, talin-L432G-, FAK-WT-, and FAK-V744G-expressing growth cones in vitro. Paired measurements were made from the same growth cones before and after the removal of AGAs, and data were normalized to the rate of adhesion assembly and duration in normal culture conditions. Statistical comparisons were made within the same growth cones before and after the removal of AGAs within each group. For each group, $n>110$ individual adhesions and $n>10$ growth cones. ${ }^{*} p<0.05$, Mann-Whitney $U$ test or Student's $t$ test.

duration. Furthermore, we concluded that calpain activity inhibits integrin-dependent adhesion signaling and point contact cycling. While previous studies have linked calpain and Src tyrosine kinase signaling in the growth cones (Robles et al., 2003), this is the first study to demonstrate that calpain regulates specific aspects of point contact adhesion dynamics in growth cones.

\section{Calpain differentially regulates adhesion assembly} and disassembly through proteolysis of talin and FAK

To determine how calpain-targeted proteolysis of talin and FAK (Fig. 1) regulates adhesion dynamics, we expressed mutant forms of talin (L432G) and FAK (V744G) that are resistant to calpain proteolysis. These mutants were used to assess the specific effects of the proteolysis of talin and FAK on adhesion dynamics. Both talin and FAK mutants have previously been shown to resist calpain proteolysis, while maintaining their function and proteinprotein interactions within the adhesion complex (Franco et al., 2004; Chan et al., 2010). In addition, mutant talin and FAK proteins correctly localize to point contact adhesions when expressed in neuronal growth cones (data not shown). We hypothesized that the expression of noncleavable talin would increase adhesion assembly, as talin is important for inside-out activation of integrin receptors (Tadokoro et al., 2003). To test this hypothesis, we coexpressed GFP-talin-L432G with paxillin-tdTomato to analyze the changes in adhesions dynamics when calpain was unable to cleave talin. As predicted, the expression of talin-L432G significantly increased point contact adhesion assembly by $23.6 \%$ compared with growth cones expressing wild-type talin (Fig. $4 e, f, i$ ). Next, we hypothesized that expressing the calpain-resistant FAK mutant would decrease point contact duration, as our laboratory previously showed that FAK promotes point contact turnover (Myers and Gomez, 2011). Interestingly, we found that the expression of FAK-V744G increased the frequency of point contact assembly by $30.2 \%$ and decreased point contact duration by $40.0 \%$ compared with growth cones expressing wild-type FAK (Fig. $4 g-j$ ). Finally, we found that, unlike the expression of wild-type proteins, the expression of either talin-L432G or FAKV744G blocked the effects of filopodial $\mathrm{Ca}^{2+}$ transients (-AGAs) on point contact adhesion dynamics (Fig. 5a,b). Altogether, these data suggest that $\mathrm{Ca}^{2+}$-activated calpain suppresses adhesion as- sembly through cleavage of both talin and FAK, and stabilizes adhesions through the cleavage of FAK.

Filopodial $\mathrm{Ca}^{2+}$ transients guide neuronal growth cones by calpain-mediated proteolysis of talin and FAK

We have previously shown that filopodial $\mathrm{Ca}^{2+}$ transients generated on one side of growth cones are sufficient to induce calpaindependent repulsive turning (Gomez et al., 2001; Robles et al., 2003). Here we wanted to determine whether calpain-mediated proteolysis of talin and FAK are also required for $\mathrm{Ca}^{2+}$ dependent growth cone turning. To address this question, we measured the turning responses of growth cones expressing GFP, GFP-capn1-H272A, GFP-talin-L432G, or GFP-FAK-V744G to focal release of caged $\mathrm{Ca}^{2+}$. After $45 \mathrm{~min}$, wild-type (NP-EGTAloaded) growth cones turn an average $21.7 \pm 3.4^{\circ}$ compared with unloaded control growth cones exposed to the same UV light pulses, which turn an average of $12.9 \pm 2.7^{\circ}$ (Fig. 6a,e,f). These results are consistent with previous observations of axon turning angles by caged $\mathrm{Ca}^{2+}$ release in growth cone filopodia (Gomez et al., 2001). Moreover, the response to caged $\mathrm{Ca}^{2+}$ release was abolished in growth cones expressing dominant-negative capn1$\mathrm{H} 272 \mathrm{~A}$ (Fig. 6e,f), which is consistent with previous findings using pharmacological inhibition of calpain (Robles et al., 2003). Interestingly, repulsive turning was also abolished in growth cones expressing either calpain-resistant talin-L432G (Fig. 6c,e,f) or calpain-resistant FAK-V744G (Fig. $6 d-f$ ). The expression of either mutant protein had no significant effect on the rate of axon outgrowth (Fig. $6 g$ ). These results demonstrate that preventing calpain-mediated proteolysis of just one adhesion protein is sufficient to prevent repulsive turning in $\mathrm{Ca}^{2+}$-dependent manner. This suggests that repulsive axon guidance cues may also signal through this $\mathrm{Ca}^{2+} /$ calpain to elicit their effects in vivo.

\section{Calpain-mediated proteolysis regulates Rohon-Beard peripheral axon extension in vivo}

To assess whether calpain-mediated cleavage of adhesion proteins regulates axon outgrowth in vivo, we used an RB skin preparation to quantify the growth and guidance of sensory neuron peripheral axons in Xenopus embryos. We chose these axons for in vivo analysis because they extend and branch along the basal 

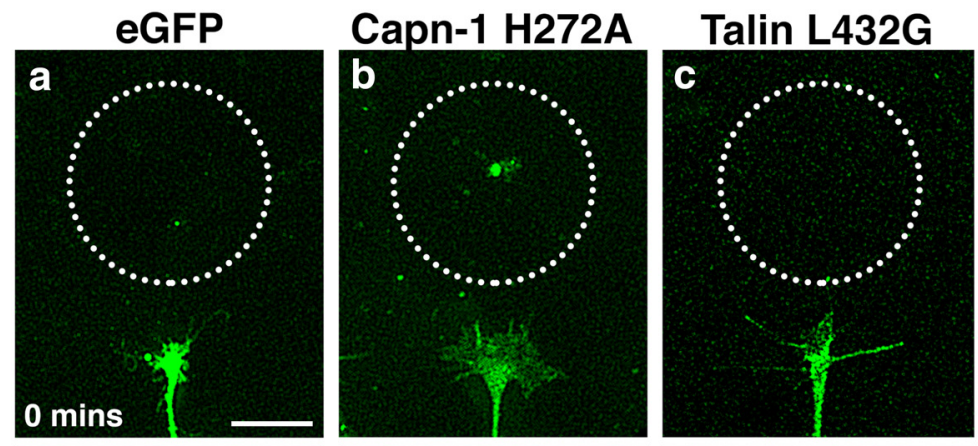

\section{FAK V744G}
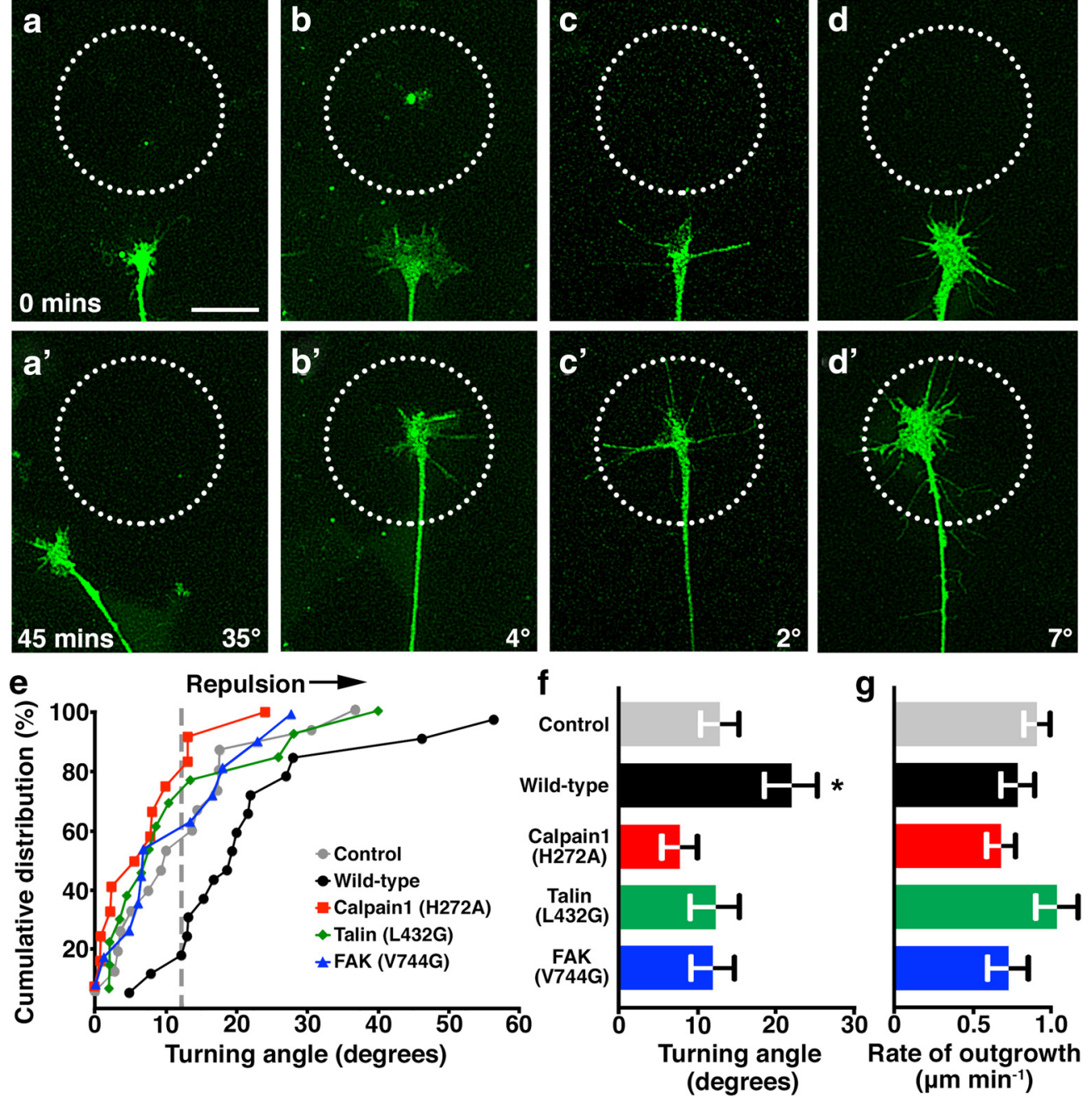

Figure 6. Stimulation of filopodial $\mathrm{Ca}^{2+}$ transients causes repulsive turning through calpain-mediated cleavage of talin and FAK. $\boldsymbol{a}, \boldsymbol{a}^{\prime}$, Two time-point images of a GFP-expressing growth cone loaded with NP-EGTA undergoing repulsive turning from an area exposed to pulsed UV light (dashed circle) at 10 s intervals. $\boldsymbol{b}$ - $\boldsymbol{d}$, Two time-point images of growth cones loaded with NP-EGTA and expressing GFP-capn1-H272A (b), GFP-talin-L432G (c), or GFP-FAK-V744G (d) show no repulsive turning to pulsed UV light after 45 min of outgrowth. $\boldsymbol{e}$, Cumulative distribution of all axon turning angles for control (no NP-EGTA, gray), NP-EGTA loaded wild-type-, capn1-H272A-, talin-L432G-, or FAK-V744G-expressing growth cones. The vertical dashed line represents the mean turning angle for the control growth cones. $\boldsymbol{f}, \boldsymbol{g}$, The mean turning angle $(\boldsymbol{f})$ and the mean rate of outgrowth $(\boldsymbol{g})$ for each condition. Scale bar, $5 \mu \mathrm{m} . n>10$ growth cones for each condition. ${ }^{*} p<0.05$, Kruskal-Wallis with Dunn's multiple-comparison test.

lamina of the skin and therefore most closely mimic our in vitro experimental conditions using a laminin substratum (Robles and Gomez, 2006; Wang et al., 2013). For these studies, we coinjected cDNA-encoding mutant constructs and GFP mRNA into one ventral blastomere at the eight-cell blastula stage (Fig. 7a; Robles and Gomez, 2006). Based on developmental fate maps, this drives expression into one side of the dorsal spinal cord and the skin (Fig. 7b). After $24 \mathrm{~h}, \mathrm{RB}$ peripheral axons were visualized with HNK-1 antibody labeling in an open-book skin preparation. This technique allowed us to compare differences between the injected side and the uninjected control side of embryos (Fig. $7 c-g$ ). When GFP alone was expressed on one side of embryos, we observed no difference in axon extension between the injected and control sides (Fig. $7 c, h$ ). In contrast, we found that peripheral axons expressing GFP-capn1-H272A were significantly longer on the injected side versus the control side (Fig. $7 f, h$ ). Interestingly, neither expression of GFP-talin-L432G (Fig. 7e) nor GFP-FAK-V744G alone (Fig. $7 f$ ) were sufficient to phenocopy the expression of dominant-negative calpain1. However, when GFP-talin-L432G and TagRFP-FAK-V744G were expressed together on the same side of the embryo, we observed a similar enhancement of axon outgrowth phenotype compared with the calpain 1 mutant embryos (Fig. $7 g, h$ ). Therefore, disruption of a single calpain substrate can affect adhesion dynamics and in vitro turning assays, but the cleavage of multiple substrates is required to affect more complex cell behaviors, such as axon outgrowth in vivo. In addition to enhanced axon lengths, we observed an increase in RB axon branching in embryos expressing dominant-negative calpain. However, we did not observe this phenotype in embryos expressing the talin and/or FAK mutants (Fig. 7i). This suggests that calpain suppresses axon branching through proteolysis of a different target, such as the actin binding protein cortactin (Mingorance-Le Meur and O'Connor, 2009).

\section{Discussion}

The goal of this study was to determine how $\mathrm{Ca}^{2+}$ signals direct calpain cleavage in filopodia to control growth cone motility. Previous work using fibroblast cell lines demonstrated that local 
a

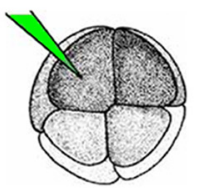

$2.5 \mathrm{hpf}$

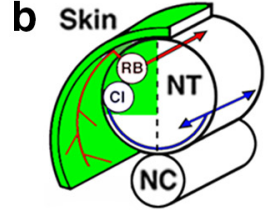

$\sim 26$ hpf
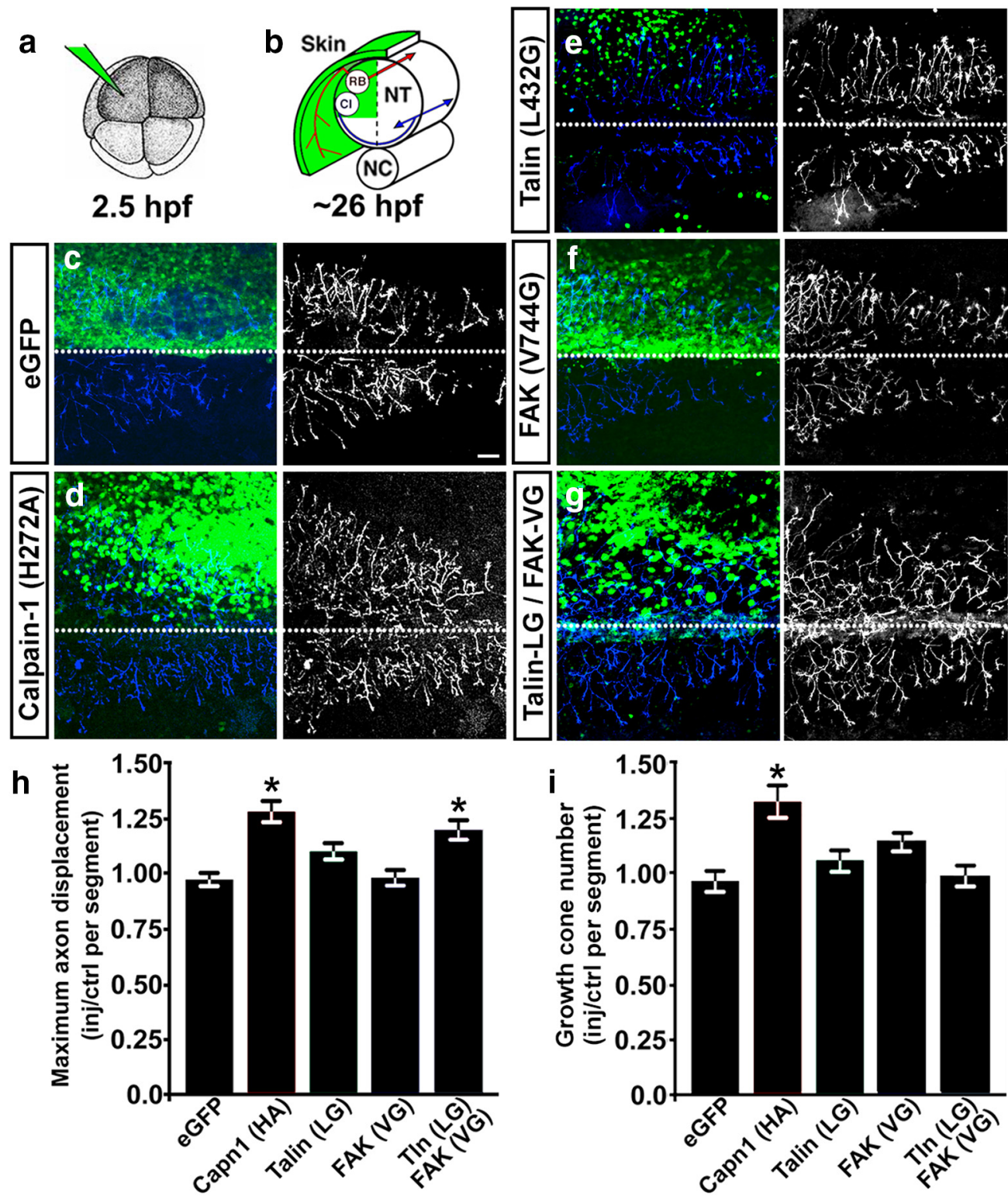

Figure 7. Inhibition of calpain activity promotes RB peripheral axon outgrowth in situ. Open-book skin preparations were used to measure axon outgrowth in situ. $\boldsymbol{a}, \boldsymbol{b}, \mathrm{GFP}, \mathrm{GFP}-\mathrm{capn} 1-\mathrm{H} 272 \mathrm{~A}$, GFP-FAK-V744G, or GFP-talin-L432G was injected in a single dark blastomere at the eight-cell stage (a) driving expression into one side of the dorsal spinal cord (b). NT, neural tube; NC, notochord. $\boldsymbol{c}-\boldsymbol{g}$, The skin of $26 \mathrm{hpf}$ embryos was removed, and preparations were labeled with NCAM to observe RB peripheral projections in the skin and flat mounted for confocal imaging. $\mathbf{c}-\boldsymbol{g}$, Representative images of HNK-1 labeling in skin expressing GFP (c), GFP-capn1-H272A (d), GFP-talin-L432G (e), GFP-FAK-V744G (f), or coexpressing GFP-talin-L432G and TagRFP-FAK-V744G (only GFP shown; $\boldsymbol{g}$ ). Dashed line represents the dorsal midline. $\boldsymbol{h}$, Quantification of the maximum axon displacement per $100 \mu \mathrm{m}$ spinal cord segment on the injected side vs control side for each condition. $\boldsymbol{i}$, Branching was quantified as the number of axon terminals (growth cones) per $100 \mu \mathrm{m}$ segment of spinal cord. Scale bar, $50 \mu \mathrm{m} . n>100$ segments and $n>10$ embryos for all conditions. ${ }^{*} p<0.05$, Mann-Whitney $U$ test.

$\mathrm{Ca}^{2+}$ and calpain signals regulate cell migration through the degradation of actin and integrin linkages (Huttenlocher et al., 1997; Franco et al., 2004; Chan et al., 2010). In agreement with these findings, we identified the adhesion proteins talin and FAK as targets of calpain proteolysis in cells isolated from the developing spinal cord (Fig. 1). We further show that calpain regulates the localization and activity of talin, FAK, and other components of integrin-based point contact adhesions of growth cones (Fig. 2). Interestingly, we find that calpain differentially regulates point contact adhesion assembly and disassembly through the cleavage of talin and FAK, respectively (Figs. 3, 4, 5). Further, we show that the cleavage of talin and FAK in growth cones is necessary for $\mathrm{Ca}^{2+}$-dependent repulsive turning (Fig. 6). These data suggest that $\mathrm{Ca}^{2+} /$ calpain activity mediates repulsive turning through asymmetrical adhesion turnover by cleaving of talin and FAK (Fig. 8). Finally, we demonstrated that proper regulation of calpain function and cleavage of talin and FAK is required for normal extension of RB peripheral axons into the developing skin (Fig. 7). These data suggest that calpain has important roles in integrin-dependent adhesion, growth cone motility, and axon guidance in vitro and in vivo.

An intriguing result from this study is the differential effects of calpain-resistant talin (L432G) and FAK (V744G) on growth cone adhesion dynamics. We find that under basal conditions, calpain-resistant FAK affected both adhesion formation and duration in growth cones, but calpain-resistant talin affected only adhesion formation (Fig. 4i,j). While calpain-resistant FAK and talin differentially affect adhesions, each is sufficient to block 

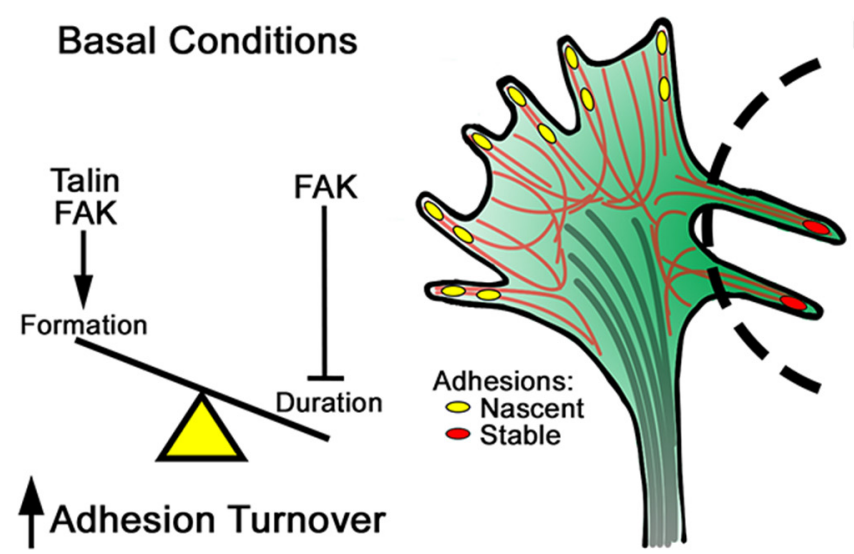

High $\mathrm{Ca}^{2+} /$ Calpain Activity

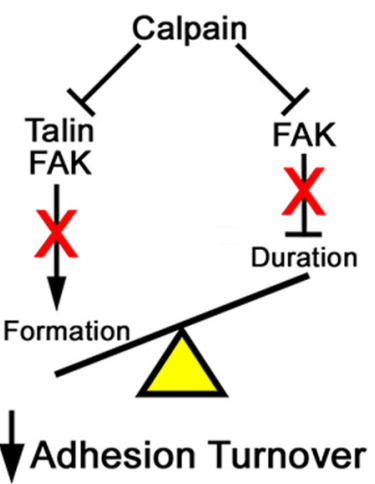

Figure 8. $\mathrm{Ca}^{2+} /$ calpain regulation of adhesion dynamics and growth cone motility. Growth cones exhibit repulsive turning from local $\mathrm{Ca}{ }^{2+}$ elevations in filopodia. Under basal conditions in our experiments, talin and FAK promote integrin-based adhesion formation, while FAK promotes the disassembly of adhesions to maintain consistent adhesion turnover. Local Ca ${ }^{2+}$ elevations (dashed lines) in response to repulsive axon guidance cues (presumed) or uncaging of $\mathrm{Ca}^{2+}$ in filopodia (this study) activates calpain. Filopodial calpain activity cleaves talin and FAK to prevent adhesion formation and turnover, and tips the balance between formation and duration that results in $\mathrm{Ca}^{2+}$-dependent repulsive turning.

repulsive turning to local $\mathrm{Ca}^{2+}$ uncaging. One explanation for this is that, under stimulated conditions, calpain-resistant talin blocks $\mathrm{Ca}^{2+}$-dependent changes on both adhesion formation and duration, which is consistent with our observations after global activation of calpain by AGA removal (Figs. $5 a, b, 8)$. Talin may affect adhesion duration indirectly by binding FAK, which may be disrupted by calpain only under stimulated conditions. However, it is important to note that due to the limitations of imaging the precise spatiotemporal dynamics of growth cone adhesions, we could be missing some subtle changes in growth cone adhesion dynamics induced by the expression of talin and FAK mutants. For example, better temporal resolution would allow us to more accurately calculate adhesion kinetics, such as rate constants for assembly and disassembly of adhesions. Future advances in imaging technologies and fluorophores will likely allow us to determine such adhesion parameters and clarify our working model for growth cone adhesion dynamics.

A major open cell biological question is whether calpain proteolysis leads to degradation or modulation of specific target proteins. Unlike degradative proteases, analysis of $>100$ substrate target sequences of calpain proteolysis did not reveal a clear consensus sequence (Tompa et al., 2004). This led to the hypothesis that calpain proteolysis is based on secondary and tertiary structures of target proteins, as calpain commonly cleaves proteins between modulatory domains, leading many to suggest that calpain modulates protein function rather than degrading proteins (Franco and Huttenlocher, 2005). For example, the head domain of talin is important for the activation of integrins during adhesion assembly (Calderwood et al., 1999), and studies using cellfree in vitro assays show that the talin head domain alone has a sixfold higher binding affinity for $\beta$-integrins compared with full-length talin (Yan et al., 2001). Furthermore, structural evidence suggests that full-length talin auto-inhibits the talin head domain, preventing binding and activation of integrins (Goksoy et al., 2008; Saltel et al., 2009). Therefore, it is possible that calpain proteolysis activates talin function by relieving talin auto-inhibition. Recent evidence in non-neuronal cells suggests that the talin head proteolytic fragment is essential for adhesion assembly, cell spreading, and membrane protrusion. However, in basal cell conditions the talin head fragment is rapidly ubiquitinated and degraded by the E3 ubiquitin ligase Smurf1, and only during specific cellsignaling events is Smurf1 blocked from ubiquitinating talin proteolytic fragments (Huang et al., 2009). A similar mechanism may occur in developing axons, as Smurfl activity is modulated in the presence of extracellular axon guidance cues, such as BDNF (Cheng et al., 2011).

Although less well studied, calpain proteolysis of FAK may also produce functional protein fragments. Calpain cleaves FAK between the kinase and focal adhesion targeting (FAT) domain, leaving the kinase domain functional but without proper localization (Fig. 1c). The FAT domain fragment resembles the endogenous dominant-negative version of FAK known as FAK-related nonkinase (FRNK; Chan et al., 2010). Interestingly, overexpression of FRNK reduces point contact adhesion cycling in neuronal growth cones (Myers and Gomez, 2011), prevents the response to attractive guidance cues ( $\mathrm{Li}$ et al., 2004), and modulates both axon extension and branching in vivo (Rico et al., 2004; Robles and Gomez, 2006). In contrast, the expression of calpainresistant talin and FAK did not prevent axon extension, rather it enhanced outgrowth in vivo, which is consistent with the notion that calpain normally suppresses motility by the generation of inhibitory protein fragments (Fig. $7 e-h$ ). Importantly, our biochemical analyses suggest that the proteolytic fragments of talin and FAK are rapidly degraded, as they are almost completely lost after $30 \mathrm{~min}$ of calpain inhibition (Fig. 1d,e). The regulation of fragment degradation may provide another level of control of growth cone motility downstream of guidance cues.

Another open question is how calpain 1 and calpain2 function differs in neuronal growth cones. The experiments in this study do not distinguish between the two subunits, but these key calpain family members may have different or even opposite effects on neuronal signaling, as described previously for synaptic plasticity (for review, see Baudry and Bi, 2016). Distinct functional effects of specific calpain subunits may result from differences in their proteolytic targets. In addition, calpain 1 and calpain 2 are activated by low $(5-10 \mu \mathrm{M})$ and high $(0.2-0.5 \mathrm{~mm})$ concentrations of $\mathrm{Ca}^{2+}$, respectively. Therefore, it is possible that calpain 1 may mediate the cleavage of talin and FAK under basal conditions (low $\mathrm{Ca}^{2+}$ activity), while calpain 2 may only be active under stimulated conditions (high $\mathrm{Ca}^{2+}$ activity). These possible differences between upstream and downstream calpain pathways suggest that calpain signaling in growth cones is more complex than previously described, which provides interesting open questions for future studies.

Many axon guidance cues may signal through the modulation of calpain activity to regulate growth cone motility and axon 
guidance. Some repulsive guidance cues, such as Slit-2 and myelin-associated glycoprotein, affect motility by activating $\mathrm{Ca}^{2+}$ influx through plasma membrane ion channels (Henley et al., 2004; Guan et al., 2007), which may activate calpain downstream. Interestingly, our previous work suggests that Slit modulates point contact adhesion dynamics in a manner that is very similar to that of calpain activation (Fig. 2; Myers et al., 2012). In addition, while Semaphorin 3A (Sema3A) is considered a $\mathrm{Ca}^{2+}$. independent repulsive axon guidance cue (Song et al., 1998), it does activate calpain through phosphorylation by MAPK and not $\mathrm{Ca}^{2+}$ influx (Qin et al., 2010). Attractive axon guidance cues may also signal through calpain by suppressing rather than promoting calpain activity. For example, recent work demonstrated that precrossing spinal commissural interneurons (CIs) exhibit high calpain activity, which is reduced in response to the midline GDNF in postcrossing CIs (Nawabi et al., 2010; Charoy et al., 2012). At the midline, calpain is thought to act on guidance cue receptors to modulate responsiveness to particular environmental cues (Charoy et al., 2012). In addition, calpain proteolysis of adhesion proteins may be modulated in response to growthpromoting guidance cues. For example, the inhibition of calpain leads to similar changes in point contact adhesion cycling, as has been observed in response to BDNF (Myers and Gomez, 2011). Netrin-1 also regulates growth cone motility through the regulation of adhesion signaling (Li et al., 2004; Liu et al., 2004; Ren et al., 2004). Interestingly, both BDNF and Netrin regulate growth cone behavior by modulating $\mathrm{Ca}^{2+}$ signaling via TRPC channels (Li et al., 2005; Shim et al., 2005; Wang and Poo, 2005). However, this is counterintuitive, since we recently showed that $\mathrm{Ca}^{2+}$ influx through TRPC1-containing channels activates calpain (Kerstein et al., 2013). One explanation for this discrepancy may be cyclic nucleotide signaling, which is known to act in parallel with $\mathrm{Ca}^{2+}$ signals. Previous studies have shown that cAMP can act as a molecular switch between $\mathrm{Ca}^{2+}$ effectors CaMKII and calcineurin to control attractive and repulsive turning, respectively (Song et al., 1998; Wen et al., 2004). In addition, cAMP/protein kinase A can directly inhibit calpain function by phosphorylation at S369 (Shiraha et al., 2002). Therefore, while $\mathrm{Ca}^{2+}$ activates all of these signaling pathways, differential signals may arise through cyclic nucleotide-dependent regulation of the $\mathrm{Ca}^{2+}$ effectors calcineurin, CaMKII, and calpain.

In addition to chemical guidance cues, mechanical cues may also regulate axon outgrowth and guidance by $\mathrm{Ca}^{2+}$ and calpain signaling. Recently, we hypothesized that $\mathrm{Ca}^{2+}$ signals may provide homeostatic feedback to point contact adhesions (Kerstein et al., 2015). For example, filopodial $\mathrm{Ca}^{2+}$ transients and mechanosensitive channels are modulated by ECM substratum rigidity and regulate axon outgrowth and guidance through $\mathrm{Ca}^{2+}$ dependent activation of calpain (Kerstein et al., 2013). Furthermore, we demonstrated that filopodial $\mathrm{Ca}^{2+}$ transients inhibit point contact adhesion dynamics via calpain-mediated proteolysis of talin and FAK (Figs. 4, 5, 6). These new data suggest a mechanical inhibitory feedback mechanism among integrin, $\mathrm{Ca}^{2+}$, calpain, and point contact adhesion proteins. This mechanism in growth cones may also affect axon extension and guidance in vivo, because RB peripheral axon outgrowth is strongly modulated by perturbations to calpain proteolysis of adhesion proteins (Fig. 7). Our model suggests that $\mathrm{Ca}^{2+}$ and calpain inhibit normal adhesion dynamics to regulate axon outgrowth and repulsive turning (Fig. 8). However, while our model fits the experimental conditions presented in this study, it is likely that the functional relevance of this mechanism varies depending on the environmental conditions in vitro and in vivo.

\section{References}

Baudry M, Bi X (2016) Calpain-1 and Calpain-2: the ying and yang of synaptic plasticity and neurodegeneration. Trends Neurosci 39:235-245. CrossRef Medline

Bechara A, Nawabi H, Moret F, Yaron A, Weaver E, Bozon M, Abouzid K, Guan JL, Tessier-Lavigne M, Lemmon V, Castellani V (2008) FAKMAPK-dependent adhesion disassembly downstream of L1 contributes to semaphorin3A-induced collapse. EMBO J 27:1549-1562. CrossRef Medline

Calderwood DA, Zent R, Grant R, Rees DJ, Hynes RO, Ginsberg MH (1999) The Talin head domain binds to integrin beta subunit cytoplasmic tails and regulates integrin activation. J Biol Chem 274:28071-28074. CrossRef Medline

Chan KT, Bennin DA, Huttenlocher A (2010) Regulation of adhesion dynamics by calpain-mediated proteolysis of focal adhesion kinase (FAK). J Biol Chem 285:11418-11426. CrossRef Medline

Charoy C, Nawabi H, Reynaud F, Derrington E, Bozon M, Wright K, Falk J, Helmbacher F, Kindbeiter K, Castellani V (2012) gdnf activates midline repulsion by Semaphorin3B via NCAM during commissural axon guidance. Neuron 75:1051-1066. CrossRef Medline

Cheng PL, Lu H, Shelly M, Gao H, Poo MM (2011) Phosphorylation of E3 ligase Smurf1 switches its substrate preference in support of axon development. Neuron 69:231-243. CrossRef Medline

Dent EW, Meiri KF (1992) GAP-43 phosphorylation is dynamically regulated in individual growth cones. J Neurobiol 23:1037-1053. CrossRef Medline

Dent EW, Gupton SL, Gertler FB (2011) The growth cone cytoskeleton in axon outgrowth and guidance. Cold Spring Harb Perspect Biol 3:a001800. CrossRef Medline

Franco SJ, Huttenlocher A (2005) Regulating cell migration: calpains make the cut. J Cell Sci 118:3829-3838. CrossRef Medline

Franco SJ, Rodgers MA, Perrin BJ, Han J, Bennin DA, Critchley DR, Huttenlocher A (2004) Calpain-mediated proteolysis of talin regulates adhesion dynamics. Nat Cell Biol 6:977-983. CrossRef Medline

Goksoy E, Ma YQ, Wang X, Kong X, Perera D, Plow EF, Qin J (2008) Structural basis for the autoinhibition of talin in regulating integrin activation. Mol Cell 31:124-133. CrossRef Medline

Gomez TM, Robles E, Poo M, Spitzer NC (2001) Filopodial calcium transients promote substrate-dependent growth cone turning. Science 291: 1983-1987. CrossRef Medline

Gómez TM, Harrigan D, Henley J, Robles E (2003) Working with Xenopus spinal neurons in live cell culture. Methods Cell Biol 71:129-156. CrossRef Medline

Guan CB, Xu HT, Jin M, Yuan XB, Poo MM (2007) Long-range Ca2+ signaling from growth cone to soma mediates reversal of neuronal migration induced by slit-2. Cell 129:385-395. CrossRef Medline

Henley JR, Huang KH, Wang D, Poo MM (2004) Calcium mediates bidirectional growth cone turning induced by myelin-associated glycoprotein. Neuron 44:909-916. CrossRef Medline

Huang C, Rajfur Z, Yousefi N, Chen Z, Jacobson K, Ginsberg MH (2009) Talin phosphorylation by Cdk5 regulates Smurf1-mediated talin head ubiquitylation and cell migration. Nat Cell Biol 11:624-630. CrossRef Medline

Huttenlocher A, Palecek SP, Lu Q, Zhang W, Mellgren RL, Lauffenburger DA, Ginsberg MH, Horwitz AF (1997) Regulation of cell migration by the calcium-dependent protease calpain. J Biol Chem 272:32719-32722. CrossRef Medline

Kerstein PC, Jacques-Fricke BT, Rengifo J, Mogen BJ, Williams JC, Gottlieb PA, Sachs F, Gomez TM (2013) Mechanosensitive TRPC1 channels promote calpain proteolysis of talin to regulate spinal axon outgrowth. J Neurosci 33:273-285. CrossRef Medline

Kerstein PC, Nichol RH IV, Gomez TM (2015) Mechanochemical regulation of growth cone motility. Front Cell Neurosci 9:244. CrossRef Medline

Kulkarni S, Saido TC, Suzuki K, Fox JE (1999) Calpain mediates integrininduced signaling at a point upstream of Rho family members. J Biol Chem 274:21265-21275. CrossRef Medline

Laukaitis CM, Webb DJ, Donais K, Horwitz AF (2001) Differential dynamics of alpha 5 integrin, paxillin, and alpha-actinin during formation and disassembly of adhesions in migrating cells. J Cell Biol 153:1427-1440. CrossRef Medline

Li W, Lee J, Vikis HG, Lee SH, Liu G, Aurandt J, Shen TL, Fearon ER, Guan JL, 
Han M, Rao Y, Hong K, Guan KL (2004) Activation of FAK and Src are receptor-proximal events required for netrin signaling. Nat Neurosci 7:1213-1221. CrossRef Medline

Li Y, Jia YC, Cui K, Li N, Zheng ZY, Wang YZ, Yuan XB (2005) Essential role of TRPC channels in the guidance of nerve growth cones by brain-derived neurotrophic factor. Nature 434:894-898. CrossRef Medline

Liu G, Beggs H, Jürgensen C, Park HT, Tang H, Gorski J, Jones KR, Reichardt LF, Wu J, Rao Y (2004) Netrin requires focal adhesion kinase and Src family kinases for axon outgrowth and attraction. Nat Neurosci 7:12221232. CrossRef Medline

Lowery LA, Van Vactor D (2009) The trip of the tip: understanding the growth cone machinery. Nat Rev Mol Cell Biol 10:332-343. CrossRef Medline

Mingorance-Le Meur A, O'Connor TP (2009) Neurite consolidation is an active process requiring constant repression of protrusive activity. EMBO J 28:248-260. CrossRef Medline

Moon MS, Gomez TM (2010) Balanced Vav2 GEF activity regulates neurite outgrowth and branching in vitro and in vivo. Mol Cell Neurosci 44:118128. CrossRef Medline

Myers JP, Gomez TM (2011) Focal adhesion kinase promotes integrin adhesion dynamics necessary for chemotropic turning of nerve growth cones. J Neurosci 31:13585-13595. CrossRef Medline

Myers JP, Santiago-Medina M, Gomez TM (2011) Regulation of axonal outgrowth and pathfinding by integrin-ECM interactions. Dev Neurobiol 71:901-923. CrossRef Medline

Myers JP, Robles E, Ducharme-Smith A, Gomez TM (2012) Focal adhesion kinase modulates Cdc42 activity downstream of positive and negative axon guidance cues. J Cell Sci 125:2918-2929. CrossRef Medline

Nawabi H, Briançon-Marjollet A, Clark C, Sanyas I, Takamatsu H, Okuno T, Kumanogoh A, Bozon M, Takeshima K, Yoshida Y, Moret F, Abouzid K, Castellani V (2010) A midline switch of receptor processing regulates commissural axon guidance in vertebrates. Genes Dev 24:396-410. CrossRef Medline

Nichol RH IV, Hagen KM, Lumbard DC, Dent EW, Gómez TM (2016) Guidance of axons by local coupling of retrograde flow to point contact adhesions. J Neurosci 36:2267-2282. CrossRef Medline

Nieuwkoop PD, Faber J (1994) Normal table of Xenopus laevis (Daudin). New York: Garland.

Qin Q, Liao G, Baudry M, Bi X (2010) Role of calpain-mediated p53 truncation in semaphorin 3A-induced axonal growth regulation. Proc Natl Acad Sci U S A 107:13883-13887. CrossRef Medline

Ren XR, Ming GL, Xie Y, Hong Y, Sun DM, Zhao ZQ, Feng Z, Wang Q, Shim S, Chen ZF, Song HJ, Mei L, Xiong WC (2004) Focal adhesion kinase in netrin-1 signaling. Nat Neurosci 7:1204-1212. CrossRef Medline

Rico B, Beggs HE, Schahin-Reed D, Kimes N, Schmidt A, Reichardt LF (2004) Control of axonal branching and synapse formation by focal adhesion kinase. Nat Neurosci 7:1059-1069. CrossRef Medline

Robles E, Gomez TM (2006) Focal adhesion kinase signaling at sites of integrin-mediated adhesion controls axon pathfinding. Nat Neurosci 9:1274-1283. CrossRef Medline

Robles E, Huttenlocher A, Gomez TM (2003) Filopodial calcium transients regulate growth cone motility and guidance through local activation of calpain. Neuron 38:597-609. CrossRef Medline

Robles E, Woo S, Gomez TM (2005) Src-dependent tyrosine phosphorylation at the tips of growth cone filopodia promotes extension. J Neurosci 25:7669-7681. CrossRef Medline
Saltel F, Mortier E, Hytönen VP, Jacquier MC, Zimmermann P, Vogel V, Liu W, Wehrle-Haller B (2009) New PI(4,5)P2- and membrane proximal integrin-binding motifs in the talin head control beta3-integrin clustering. J Cell Biol 187:715-731. CrossRef Medline

Santiago-Medina M, Gregus KA, Gomez TM (2013) PAK-PIX interactions regulate adhesion dynamics and membrane protrusion to control neurite outgrowth. J Cell Sci 126:1122-1133. CrossRef Medline

Santiago-Medina M, Myers JP, Gomez TM (2011) Imaging adhesion and signaling dynamics in Xenopus laevis growth cones. Dev Neurobiol 72 : 585-599. CrossRef Medline

Shim S, Goh EL, Ge S, Sailor K, Yuan JP, Roderick HL, Bootman MD, Worley PF, Song H, Ming GL (2005) XTRPC1-dependent chemotropic guidance of neuronal growth cones. Nat Neurosci 8:730-735. CrossRef Medline

Shiraha H, Glading A, Chou J, Jia Z, Wells A (2002) Activation of m-calpain (calpain II) by epidermal growth factor is limited by protein kinase A phosphorylation of m-calpain. Mol Cell Biol 22:2716-2727. CrossRef Medline

Song H, Ming G, He Z, Lehmann M, McKerracher L, Tessier-Lavigne M, Poo M (1998) Conversion of neuronal growth cone responses from repulsion to attraction by cyclic nucleotides. Science 281:1515-1518. CrossRef Medline

Tadokoro S, Shattil SJ, Eto K, Tai V, Liddington RC, de Pereda JM, Ginsberg MH, Calderwood DA (2003) Talin binding to integrin beta tails: a final common step in integrin activation. Science 302:103-106. CrossRef Medline

Thévenaz P, Unser M (2007) User-friendly semiautomated assembly of accurate image mosaics in microscopy. Microsc Res Tech 70:135-146. CrossRef Medline

Tompa P, Buzder-Lantos P, Tantos A, Farkas A, Szilágyi A, Bánóczi Z, Hudecz F, Friedrich P (2004) On the sequential determinants of calpain cleavage. J Biol Chem 279:20775-20785. CrossRef Medline

Vitriol EA, Zheng JQ (2012) Growth cone travel in space and time: the cellular ensemble of cytoskeleton, adhesion, and membrane. Neuron 73: 1068-1081. CrossRef Medline

Wang F, Julien DP, Sagasti A (2013) Journey to the skin: somatosensory peripheral axon guidance and morphogenesis. Cell Adh Migr 7:388-394. CrossRef Medline

Wang GX, Poo MM (2005) Requirement of TRPC channels in netrin-1induced chemotropic turning of nerve growth cones. Nature 434:898 904. CrossRef Medline

Wei C, Wang X, Chen M, Ouyang K, Song LS, Cheng H (2009) Calcium flickers steer cell migration. Nature 457:901-905. CrossRef Medline

Wen Z, Guirland C, Ming GL, Zheng JQ (2004) A CaMKII/calcineurin switch controls the direction of $\mathrm{Ca}(2+)$-dependent growth cone guidance. Neuron 43:835-846. CrossRef Medline

Woo S, Gomez TM (2006) Racl and RhoA promote neurite outgrowth through formation and stabilization of growth cone point contacts. J Neurosci 26:1418-1428. CrossRef Medline

Woo S, Rowan DJ, Gomez TM (2009) Retinotopic mapping requires focal adhesion kinase-mediated regulation of growth cone adhesion. J Neurosci 29:13981-13991. CrossRef Medline

Yan B, Calderwood DA, Yaspan B, Ginsberg MH (2001) Calpain cleavage promotes talin binding to the beta 3 integrin cytoplasmic domain. J Biol Chem 276:28164-28170. CrossRef Medline 